\title{
Leveraging Classification of Brain Tumour using Deep Learning Architectures
}

\author{
Karunya K, Karpagam G R and Swathi $\mathbf{J}^{1}$ \\ \{karunyakandimuthu@gmail.com, grk.cse@psgtech.ac.in, jsw.cse@psgtech.ac.in $\left.{ }^{1}\right\}$ \\ Department of Computer Science and Engineering, PSG College of Technology, \\ Coimbatore, Tamilnadu, 641004,India
}

\begin{abstract}
Despite advances in human intellect and biomedical in the last few decades, people continue to suffer from various cancers due to their volatile nature. This disease is still a major issue for the entire humanity. Brain tumour is one of the most crucial and serious illnesses. Oftheentire primary central nervous system tumours, Brain tumorsmake up 85 to $90 \%$. It isestimatedthat 18,600 adults, including 8,100 women and 10,500 men, will die of primary cancerous tumors of the brain and centralnervoussystem tumors this year. Among the children of various age groups also it is seen as one of the most crucial cancers. Thus, accurate and timely handling of this disease is decisive. In order to speed up the process of brain tumour detection (augmented with accuracy, reliability and experience)Deep learning models can be used.To efficiently diagnose brain tumour kinds and compare classification performance, the proposed work makes optimal use of a newly modelled Convolutional Neural Network and ResNet 50,a pre-trained network.
\end{abstract}

Keywords: Brain tumour, deep Learning, Classification, Comparison, CNN, ResNet50.

\section{Introduction}

The need for novel healthcare solutions and ongoing efforts tounderstand the biological basis of pathologies havefueled extensive life science research over the past two centuries. Machine Learning plays a major role in classification of images, recommendation of videos, and mining information from the textual data etc.

Deep learning is one of the most extensively utilized machine learning algorithms in these applications. Representational Learning is another name for DL (RL). The unpredictability of the increase in data retrieval skills, as well as the astonishing advances in hardware technologies, such as High Performance Computing (HPC), has resulted in the publication of new studies in deep learning on a regular basis.DL is a variant of the customary neural network that outpaces its forerunners. In addition, DL creates layered learning models by combining transformations and graphics technologies. In a range of applications, together withaudio processing, computing the visual representation, and Natural Language Processing, more recently developed DL approaches have performed admirably. The ML method is strongly reliant on the input data representation's integrity, and it has been shown that a good data representation outperforms a bad data representation.For many years, feature engineering has been a prominent research topic in machine learning, influencing various research papers. This method aims to generate features from data that is raw. It is also highly subjectspecific and recurrently necessitates significant the effort of the human community. Different 
types of features,were familiarized and matched in the background of computer vision. When a new function is created and accepted, it turn out to be a new research focus that has been pursued for very long years. The mining of the significant features is carried out by the DL algorithms automatically. Researchers are encouraged to extract discriminatory traits with the least amount of human work and field knowledge possible. The earliest levels of these algorithms extract low-level features, whereas the last layers extract high-level features, resulting in a multilayered data representation architecture. This architecture, that is capable of modelling the process that occurs in the central sensory regions of the brain of a human, was inspired by artificial intelligence (AI). Using numerous scenarios, the human brain can automatically derive the representation from the data.More exactly, categorization of objectshappens as a result of this process. This process mimics the way the human brain functions. As a result, the primary benefit of the DL is highlighted, which is one of the most important study trends in the DL field due to its widespread popularity. Concepts, architectures, difficulties, applications, computing tools, and an evolution matrix are the main concepts. One of the most prevalent and generally used DL networks is the Convolutional Neural Network (CNN). Today, DL is quite popular because of CNN. The key advantage of Convolutional Neural Network over its precursors is that it automatically detects the imperative aspects it has used the most without the need for human interference. Also CNN does a detailed examination of the image which makesCNN outpacesthe other whole image matching algorithms in similarity detection.CNN magnificently down samples the image using neighboring pixel information, followed by convolution and finally a prediction layer [16].This study was created with the goal of covering the most significant aspects of DL, such as open challenges, healthcare applications, and computing tools.

\section{Concepts}

For the proper understanding of the readers, the terms and terminologies are described below.

Preparation: In Brain Tumour Classification System, the images should be named in such a way it is easy to navigate through the directories and folders to extract the training and test image files. Once the navigation to the right folder is done and the image is accessed, it is resized and is passed for further processing.

Classification: Once the training is done, the trained model is used for predicting the class label of the test images depicting the type of brain tumour.

Convolution Neural Network: A convolutional neural network termed as CNN or ConvNet is a popular model of deep learning which is proficient of recording the input images, assigningprominence to multiple characteristics or different objects observed in the image, and distinguishing between them. From the input image fed to the network, the convolution technique abstracts features with high-level including edges in the image. ConvNets need not be limited to asinglelayer of convolution. Usually, the first layer of convolution is responsible for extracting the low-level features which includes color and alignment of thegradient. Withadding few more layers, the networks adapts to features of 
high-levelfunctionsandgives us a network that fully understands the images in the data set,muchlike we would.

Pre-trained Models: A model that has self-reliantly learned extrapolative associations from training data. It's a model developed by someone else to handle a comparable challenge. Instead of starting from scrape to solve a comparable problem, start with a model that has already been trained on a different problem.

\section{Related Works}

Literature surveys help to discover what statistical knowledge exists related to the research topic. It helps to find gaps in existing research so that new original ideas can be generated. The relevance to the proposed idea can be justified. Few references are made and the content of the paper works are summarized below.

The purpose of reference [1] was to reduce the overfitting problem and increase classification accuracy. In the system proposed, a novel Convolutional Neural Network with enhanced loss function and regularization is used. The suggested system has three steps: preprocessing, classification, and extraction. The pre-processing stage aims at reducing the noise observed by maintaining tumour edges in the collected MRI pictures are maintained. At this stage, a median filter of $3 \times 3$ dimension was utilized as an alternative of the traditional filters.Using the filter employed improves the contrast and the image quality. During the classification stage, ELM-LRF (Extreme Machine Learning- Local Receptive Field) is used. The ELM-LRF structure is made up of two parts: first part with a pooling and a convolution layer, and in the second part the features extracted are merged .Instead of employing the sigmoid function for classification, the proposed method uses a enhanced loss function in the convolution layer. When compared to the old approach, the proposed method obtained a 98.99 percent accuracy and significantly reduced the processing time.

Reference [2] examines the impact of several pre-trained word embedding models for sentiment analysis on drug evaluations and shows many model combinations, such as LSTM which stands for long short-term memoryand $\mathrm{CNN}$ which stands for Convolutional Neural Network. It's also being looked into using a Bidirectional Encoder Representations from Transformers (BERT) and Bidirectional LSTM for sentiment analysis of medicine reviews. Precision, recall, and the F1 metric are all common evaluation metrics.According to the results, LSTM combines with CNN produces the highest value for macro-F1 and microF1.The work proposed aims in integrating language representation models in addition to variation auto encoders and for semi supervised techniques adversarial networks can be employed in future work because of the rising use of data representation models for many problems of classification. In addition, to increase the refinement of the techniques used, investigating the usage of semantic features in conjunction with the contextual ones is done.

Reference [3] presented an EEG-based diagnosis system that might be used to diagnose epilepsy and autism, two neurological illnesses. The study presents a novel dynamic filtering approach that identifies and pre-processes the most instructive sub-bands related to specific neurologicalailment using Finite and Infinite Impulse Response filters, as well as Recurrent Neural Networks with a Gated-Recurrent Unit.For epilepsy, the suggested diagnosis approach 
obtains a classification accuracy of 100 percent, while for autism, it achieves a classification accuracy of 99.5 percent.

Reference [4] proposes an enhanced hybrid deep learning model for detecting fake news by combining Recurrent and Convolutional Neural Networks.The suggested model takes advantage of the CNN's capacity to extract local characteristics as well as the LSTM's ability to learn the dependencies of long-term. In future more complex neural network models are planned to be explored.

A novel CNN model is presented in Reference [5] for feature extraction from the images of the retinal fundus to enhance the performance of the classification. In the system proposed, the input feed for different classifiers of machine learning is taken from the features extracted from the CNN. Using images from the generic IDRiD, MESSIDOR, and KAGGLE datasets, the model is tested using multiple classifiers namely Naïve Bayes, Support Vector Machine, Random Forest, Adaboostand finally J48. Different accuracy parameters like Kappa-score or the K-score, precision, recall, False Positive Rate, for different classifiers used are compared to determine the efficacy of the classifier.The suggested feature extraction strategy pooled with the J48 outpaces theother supplementary classifiers on different datasets used, with an average accuracy of 99.89 percent for binary classification and 99.59 percent for multiclass classification.The main contribution of the work is a considerable reduction in CNN model parameters, allowing for enhancingthe accuracy of classification. With modest tweaks, the same network could be used to detect other retinal diseases in the future.

At the time of capture, rician noise can corrupt magnetic resonance images (MRI). This type of noise generally degrades the ability of a physician or asystem automated to diagnose sickness. As a result, in pre-processing phase rician noise must be removed from MRI images. References [6] proposed CNN-DMRI, a new Convolutional Neural Network that helps in denoising MRI data. The proposed CNN outperforms previous techniques, according to the results of the comparison research.

In reference [7], various convolution neural network architectures that are deep were presented to do an effective feature extraction from the X-ray images of the chest and further perform a classification task to determine if a person is suffering frompneumonia. Research showed the two different CNN designs, one utilizing a dropout layer and the other without the dropout layer. The work is intended to apply other techniques for optimization along with data augmentation methods in the future to enhance the accuracy of the classification. It is also intended for testing the effects of batch normalisation and early stopping as an alternative of the dropout layer in avoiding overfitting.

According to reference [8], the pre-processing stage utilizes the speech improvement strategy of EMDH to enhance the speech quality of dysarthria. From the EMDH-processed speech, the cepstral coefficients of Mel frequency are extracted and sent into a CNN-based recognizer as input characteristics. The findings imply that the CNN is capable of retrieving latent characteristics of dysarthria speech and that it may be trained faster with fewer data. To improve dysarthria speech, Hurst-based mode selection andempirical mode decomposition was employed as a pre-processing phase.Other robust and strong acoustic properties, as well as deep learning network with long-term, memorywill be researched in the future. 
With the growing number of biological images, determining how to efficiently identify them has become a difficult task. Convolutional Neural Networks (CNNs) show promise in such scenarios. Imagesof fixed-size as CNN input were substituted with properly images of large-sized in Reference [9], and certain modules have been substituted by Inverted Residual Block modules, which have lower computational costs and characteristics. This is done to tackle the problem of higher computation generated by this approach. The approach was tested on five different benchmark datasets, out of which two were biological imaging data which showed promise in terms of accuracy and parameter reduction. The experiment also demonstrates approach scalability, demonstrating that it may be applied to a variety of networks. The plan of the work is to find a convolution method which is more effective and trivial in the future to reduce network parameters more effectively.

On three separate data sets, reference [10] compared DSNet to many prominent algorithms and found that DSNet gets superior results while using fewer training samples. This paper presents a new CNN called DSNet that replaces the traditional convolution operation with depth wise separable convolution to perform a feature extraction of the PolSAR images effectively and also avoids the redundant features. CNN stands for Conditional Probability Network. As a result, it is unable to handle classification issues in which the training dataset's distributions differ from those of the test dataset.CNN algorithms are unlikely to perform well if both training andtesting datasets are not from the same source having different look angles and wavelengths.

Reference [11] suggested a CNN model for multi-classification of brain tumours, including meningioma, pituitary tumour, and glioma. The original image is shortened from the dimension of $512 \times 512 \times 1$ to $128 \times 128 \times 1$ in the pre-processing procedure to minimise the image's dimension. Three convolutional layers are included in the suggested design. When the filters are moved alongside the input, a $2 \mathrm{D}$ layer of convolution slides $\mathrm{K}$ number of convolutional filters which is of size $\mathrm{M}$ and $\mathrm{N}$ and computes dot product of the weights with the corresponding input. A non-saturated ReLU activation function follows each convolution layer. Drop out layer is included to minimize the overfitting, and finally a layer that is fully connected and to predict the output, a softmax layer is included.The accuracy obtained for the meningioma classification was 97.54 percent, the glioma classification was 95.81 percent, and the pituitary classification was 96.89 percent. Glioma Grade II has a 100 percent classification accuracy, glioma Grade III has a 95 percent classification accuracy, and glioma Grade IV has a 100 percent classification accuracy.

Reference [12] suggested a tweaked and tuned version ofGoogLeNet for feature extraction of brain MRI images.Along with the softmax layer in deep CNN network, the proposed research looked at the CNN features extracted on two different classifiers, including Support Vector Machine combined with an error-correcting output code and KNN with k value of 49.SVM is tolerable and works well with data with several dimensions. It can also do nonlinear classification competentlyexploiting a method known as the kernel trick, which is involved in mapping the inputs into a high-dimensional feature spaces [18].Adam was chosen as the best optimizer because to its high learning rate and parameter-specific adaptive nature The initial learning rate of Adam's optimizer was fixed to 0.0003. Accuracy of classification of brain tumour by standalone deep transfer learned model obtained is $92.3 \%$. SVM has a $97.8 \%$ accuracy on deep CNN features. KNN has a $98.0 \%$ percent accuracy on deep CNN features. 
A new method for classification ofmulti-class MRI brain tumours utilizing a convolutional neural network was proposed in reference [13]. Pre-training and fine-tuning are the two phases of the learning algorithm. Before being fed to GAN, MR pictures from both datasets go through a data augmentation process. MRI scans used are down-sampled from pixel of $512 \times 512$ to $64 \times 64$ and sent to the classifier used. The main goal of this pre-training is to overcome the problem of small datasets. The work makes use of an unsupervised pretraining method that doesn't require any labelled data, therefore any dataset pertaining to the problem can be utilized GAN architecture is used as a discriminator to pre-train the developed deep network. After the pre-training process the network was trained on the key dataset, with a Soft Max classifier. On employing the introduced split the accuracy obtained is 93.01 percent and on random split it is 95.6 percent, which is greater than all precedinginvestigation on the dataset.

Reference [14] as a source, the method described here is broken down into three phases. The high pass and median filters are chosen in the initial step to enhance the input photos. As part of the second stage, using the seed growth process the segmentation of brain tumour happens. In the final stage, the images segmented are fed into the SSAE (stack sparse auto encoder) model, which employs two hidden layers for classification, which are then concatenated with a softmax layer. Unsupervised learning is the foundation of the Auto Encoder (AE) neural. AE reconstructs images taken as and generates output images of the same size.The AE process is divided into two stages: encoder and decoder. The approach is put to the test on all of BRATS' most difficult datasets. On average, the accuracy was $100 \%$ in $2012,90 \%$ in 2012 synthetic, $95 \%$ in $2013,100 \%$ on Leader board 2013, 97 percent in 2014, and $95 \%$ in 2015.

Reference [15] developed a CNN-based integrated deep learning segmentation and grading model to enable simultaneous LGG (Lower Grade Glioma) tumour segmentation, identification, and grading using MRI data. The first pre-processing stage involves the cropping of non-tissue portions from the MRI images collected. Cropping was done on each patient's photos in three dimensions (height, breadth, and elevation — image slices).The segmentation model's tumour masks were utilized for the automation of the selection of tumour images for the purpose of grading. The $\mathrm{CNN}$ based segmentation model and U-net architecture is used in this work. The down-sampling phase contains the U-net architecture which consists of four layers: Two layers of convolution combined with ReLU and onelayer of max pooling, in case of up-sampling phase, it has four layers: instead of a layer of max pooling, a convolution transpose layer is used.Tumor detection accuracy achieved is $92 \%$ and the Tumor grading accuracy obtained is $89 \%$.

\section{Lessons Learnt}

The early detection of brain tumours has the potential to save millions of lives. The use of MRI to detect brain tumours earlier may help the patients live longer.

The proposed work context is described in Fig 1. 
Methods: A brain tumour's diagnosis can be performed using both CT (Computed Tomography) and MRI(Magnetic Resonance Imaging).Because of its greater image quality, MRI is chosen. MRI can distinguish between water, fat, muscles, and other soft tissue and has a stronger contrast for soft tissue when compared to the CT scan images.

Approaches: Classification model can be built using different approaches as Evolutionary computing especially Genetic Algorithm, Machine Learning and Deep Learning. The advantage of representation learning where the automatic deduction of features occurs and the network iseffectively fine-tuned for getting the anticipated outcome is seen in deep learning making it more effective for the image classification.

Platforms: MATLAB is more convenient in developing data presentation, and other programs performing specific tasks and the wide spread functions across multiple packages in $\mathrm{R}$ makes it slower than other platforms whereas OpenCV is much faster in execution.

\section{Case Study}

\section{Executive Summary}

The study is to examine the use of deep learning by constructing a model which will effectively perform the classification of various brain tumours from the MRI images into different classes namely
I. Meningioma,
II. Glioma,
III. Pituitary Tumour,
IV. No Tumour 


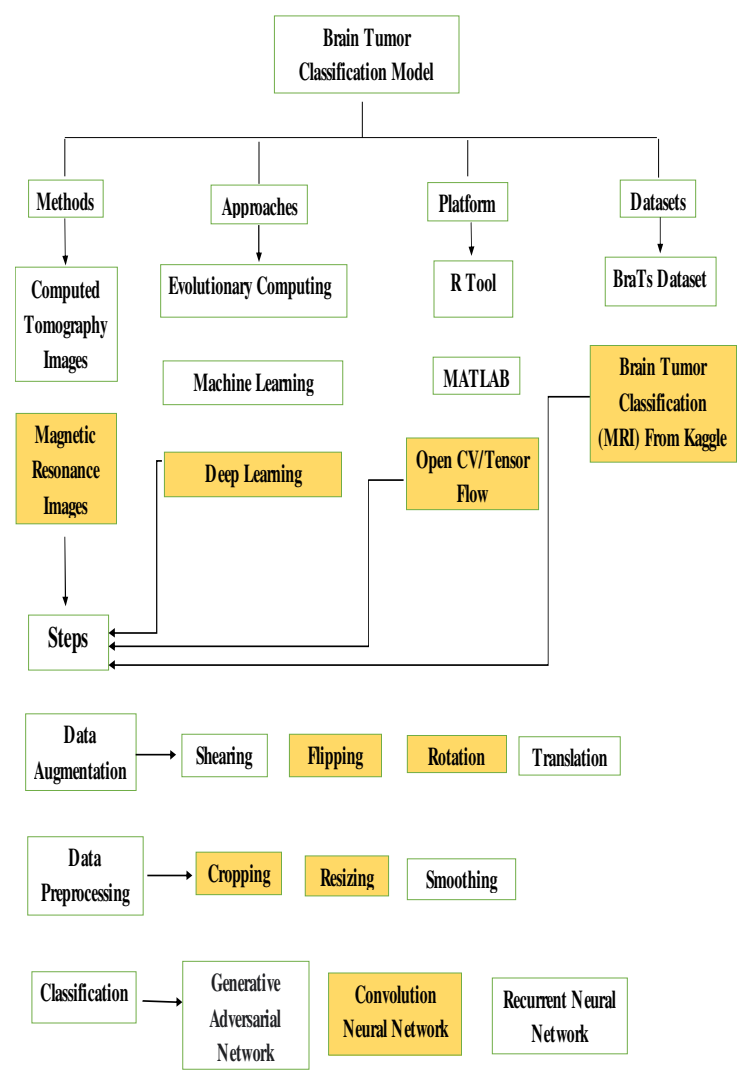

Fig 1 Context of the Research

\section{Proposed Solution}

Deep learning algorithms have proved to perform well on supervised machine learning and image classification tasks in recent studies. Convolution Neural Network is chosen for building the model that will be capable of categorising the brain tumour types since it has the advantage of automatically recognising essential aspects without the need for human supervision. In addition, $\mathrm{CNN}$ is computationally efficient. It uses specific convolution and pooling methods, as well as sharing of parameters. CNN models may now operate on any device, making them more accessible to a wider audience.

The process of using Magnetic Resonance Imaging to classify brain tumours is outlined below.

i. Data Augmentation Module

The fundamental flaw in present brain tumour classification research is that the model constructed suffers from overfitting due to the small dataset employed. Using data 
augmentation techniques, this challenge can be solved. It's a method for artificially inflating the size of the training dataset by generatingreformed versions of the training data. The deep learning neural network in Keras toolkit's ImageDataGenerator class allows you to fit models with image data augmentation.Various flips, rotation, zooms, Shifts, and other transformation pertaining to the modification of images are encompassed in transformation. To extend the amount of the training dataset, flipping and rotation will be used in the proposed work.

ii. $\quad$ Pre-processing Module

Data pre-processing in image classification is necessary to clean the image data for model input. In brain tumour classification, pre-processing is done in order to crop the part that contains only the brain of the image for an effective classification. To achieve this, cropping methodology to discover the extreme corners of the MRI images is employed.

\section{iii. $\quad$ Classification Module}

This work aims to compare the performance of two deep learning models - Convolution Neural Network and a pre-trained model Residual Network (ResNet-50).

Convolution Neural Network: A CNN with three blocks of convolution, each with a layer of max pooling layer, and a fully-connected layer with 128 units, triggered by a popular activation function, Rectified Linear Unit. In order to perform optimization, Adam optimization technique with SparseCategoricalCrossentropy in place of the loss function will be used. Finally, a drop out layer will be added to control the overfitting impact.

ResNet-50: ResNet50 consists of 48 layers of Convolution, one MaxPooling layer, and one Average Pooling layer in a ResNet variation. The network is distributed into 5 phases, each phase with a convolution and identity block. The network was made of three layers of convolution in all blocks of convolution, and three layers of convolution in every identity block. ResNet's main advantage is that it can swiftly train networks with a large number of layers while maintaining a low training error rate. It also makes it easier to outfit the vanishing gradient problem with identity mapping.

Dataset Description: On Kaggle, the dataset for brain MRI images can be found. Magnetic Resonance Imaging data is stored in this folder. In jpg format, this dataset contains 2870 training and 394 testing MRI pictures. Pituitary tumour, glioma tumour, meningioma tumour, and no tumour are among the four subfolders in the folder. These folders contain MRIs of various tumour classes.

\section{Implementation Platforms and Libraries Required}

Google Colab:It is an excellent tool for construction of deep learning models. The Jupyter notebook is hosted in the cloud platforms that avoids the installation of the software which makes the work simpler. Significant resources like TPUs and GPUs are provided by Google forprocessing irrespective of the hardware capabilities of one's own machine. With verylimited lines of code, important steps of machine learning or deep learning like importing the dataset that is collected, training the classifier built along with testing.

TensorFlow:In today's world tt is one of the most widespread and extensively used opensourceframework for building deep learning models It is composed of a large, adaptable 
network of libraries, tools and other resources that allow the developers to efficiently build and evaluate various machine learning and deep learning models in a very fast manner.

OpenCV: OpenCV is a real-time optimized Computer Vision library that includes tools, hardware, and software. It also supports model execution for both Machine Learning and Deep learning. OpenCV provides access to over 2,500 cutting-edge and traditional algorithms. It is typically used in processing different tasks of real time and delivering the output with algorithmic efficacy. It's a typical library that can be used in a variety of languages, and it's still evolving. The import cv2 command in Python imports Opencv. The 2 at the end denotes the use of the opencv2 version [18].

Keras:It is a popularly used python framework which is an open source that helps in construction and evaluation of various machine learning and deep learning models.For extensive numerical computation it includesTensorFlow and the Theano which make it possible to train a neural network models with limited code.

Scikit-learn:The sklearn library includes a number of useful machine learning and statistical modelling methods, such as dimensionality reduction, classification, clustering and regression. To leverage the data splitting into training, validation and testing sets, this library can be employed.

\section{Discussion And Conclusion}

The method of identifying brain tumour images from MRI scan images using CNN is expected to aid in more accurate diagnosis of brain tumours, potentially saving the lives of thousands of individuals. The work's future goals include developing a model that can partition or segment the tumour's region using deep learning models like U-Net, which can be trained with a small number of training examples, but it performs well in the task of image segmentation [17] which would aid surgeons in making informed surgical decisions and predicting patient survival rates.

\section{References}

[1] Mr Sunil Maharjan et al,"A Novel Enhanced Softmax Loss Function for Brain Tumour Detection using Deep Learning”,Journal of Neuroscience Methods (2019),Elsevier.

[2] Cristóbal Colón-Ruiz , Isabel Segura-Bedmar," Comparing deep learning architectures for sentiment analysis on drug reviews" in Journal of Biomedical Informatics(2020),Elsevier.

[3] Ghaith Bouallegue, Ridha Djemal, Saleh A. Alshebeili , And Hesham Aldhalaan,” A Dynamic Filtering Df-Rnn Deep-Learningbased Approach For Eeg-Based Neurological Disorders Diagnosis", Volume 8, 2020,IEEE Access.

[4] Jamal Abdul Nasir a,b , Osama Subhani Khanb , Iraklis Varlamis c, "Fake news detection: A hybrid CNN-RNN based deep learning approach", International Journal of Information Management Data Insights 1 (2021) 100007, Elsevier.

[5] Gayathri S, Varun P. Gopi, P. Palanisamy," A lightweight CNN for Diabetic Retinopathy classification from fundus images", Biomedical Signal Processing and Control(2020),Elsevier.

[6] Prasun Chandra Tripathi , Soumen Bag," CNN-DMRI: A Convolutional Neural Network for Denoising of Magnetic Resonance Images", Pattern Recognition Letters 135 (2020) 5763,Elsevier. 
[7] Harsh Sharma, 2 Jai Sethia Jain, 3 Priti Bansal, Sumit Gupta," Feature Extraction and Classification of Chest X-Ray Images Using CNN to Detect Pneumonia", 10th International Conference on Cloud Computing, Data Science \& Engineering (Confluence),IEEE.

[8] Mohammed Sidi Yakoub, Sid-ahmed Selouani, Brahim-Fares Zaidi and Asma Bouchair," Improving dysarthric speech recognition using empirical mode decomposition and convolutional neural network", Mohammed et al. EURASIP Journal on Audio, Speech, and Music Processing (2020), Springer.

[9] Jiaohua Qin, Wenyan Pan, Xuyu Xiang $\square$, Yun Tan, Guimin Hou,"A biological image classification method based on improved CNN", Ecological Informatics 58 (2020) 101093,Elsevier.

[10] Ronghua Shang, Jianghai He, Jiaming Wang, Kaiming Xu, Licheng Jiao, Rustam Stolkin ,” Dense connection and depthwise separable convolution based CNN for polarimetric SAR image classification", Knowledge-Based Systems 2019, Elsevier.

[11] Hossam H. Sultan et al," Multi-classification Of Brain Tumor Images Using Deep Neural Network", Special Section On Scalable Deep Learning For Big Data, Volume 7, 2019 ,IEEE Access.

[12] S. Deepak , P.M. Ameer,"Brain tumor classification using deep CNN features via transfer learning",Computers in Biology and Medicine 111 (2019) 103345, Elsevier.

[13] ]Navid Ghassemi, Afshin Shoeibi, Modjtaba Rouhani," Deep neural network with generative adversarial networks pre-training for brain tumor classification based on MR images", Biomedical Signal Processing and Control 57 (2020) 101678, Elsevier.

[14] Javaria Amin et al," Brain Tumor Detection By Using Stacked Autoencoders In Deep Learning", Journal Of Medical Systems (2020) 44:32,Springer Publications.

[15] Mohamed A. Naser et al," Brain Tumor Segmentation And Grading Of Lower-grade Glioma Using Deep Learning In MRI Images”, Computers In Biology And Medicine 121 (2020) 103758,Elsevier.

[16] Guruvareddiyur Rangaraju Karpagam, Abishek Ganapathy, Aadhavan Chellamuthu Kavin Raj, Saravanan Manigandan, J. R. Neeraj Julian, and S. Raaja Vignesh, "Leveraging CNN Deep Learning Model for Smart Parking", Deep Learning and Big Data for Intelligent Transportation, Studies in Computational Intelligence 945, Volume1Pages 151-174,Springer,2021.

[17] Amutha devi, Gayathri Monicka.J, "Development of fuzzy approach to predict the fetus safety and growth using AFI" Journal of super computing" Dec-2019 DOI 10.1007/s11227-019-03099-8

[18] G. R. Karpagam, A. Mukeshpandi, K. S. Sanjay Krishnan and S. Vanjinathan,” AI Powered Partially Parallelized Scheme for Multifactor Authentication", Manoj Kumar Mishra Bhabani Shankar Prasad Mishra Yashwant Singh Patel Rajiv Misra (eds) Smart Techniques for a Smarter Planet. Studies in Fuzziness and Soft Computing, vol 374. Springer (2019). 